This is a postprint version of the following published document:

Guzmán, E., San Miguel, V., Peinado, C., Ortega, F. \&

Rubio, R. G. (2010): Polyelectrolyte Multilayers

Containing Triblock Copolymers of Different Charge

Ratio. Langmuir, 26 (13), pp. 11494-11502.

"This document is the Accepted Manuscript version of a Published Work that appeared in final form in Langmuir, (C) 2010 American Chemical

Society after peer review and technical editing by the publisher.

To access the final edited and published work see http://pubs.acs.org/doi/abs/10.1021/la101043z, or see DOI: $10.1021 / 1 \mathrm{la101043z} "$ 


\title{
Polyelectrolyte Multilayers Containing Triblock Copolymers of Different Charge Ratio
}

\author{
Eduardo Guzmán, ${ }^{\dagger}$ Verónica San Miguel, ${ }^{\dagger}$ Carmen Peinado, ${ }^{*}$ Francisco Ortega,${ }^{\dagger}$ and \\ Ramón G. Rubio*,† \\ ${ }^{\dagger}$ Departamento de Química Física I, Facultad de Ciencias Quimicas, Universidad Complutense de Madrid, \\ Ciudad Universitaria s/n, 28040 Madrid, Spain, and "Instituto de Ciencia y Tecnología de Polímeros, C.S.I.C., \\ C/Juan de la Cierva 3, 28006 Madrid, Spain
}

\begin{abstract}
on the type of the amino groups, either hydrochloride or quaternized. This leads to changes in the charge density of the chains for the same content of amino groups. The growth of the multilayers has been followed using dissipative quartz crystal microbalance and ellipsometry techniques. The results show that, independently of the conditions used in the assembling, the film thickness grows linearly with the number of layers. The comparison of the thickness values obtained from D-QCM and ellipsometry has allowed us to calculate the water content of the polymer film. The analysis of the D-QCM data also provides the shear modulus, whose values are typical of a rubber-like polymer system. The analysis of the mass adsorbed calculated by the ellipsometric measurements indicated that the nature of the charge compensation mechanism is extrinsic for all the studied systems, although the degree of extrinsic compensation is strongly dependent on the copolymer used and the concentration in solution. Finally, it was found that the adsorption kinetic of the layers is bimodal for all the films built. Even though the characteristic adsorption times depend on the specific copolymer used, no dependence on the number of layers has been found for a given multilayer.
\end{abstract}

Multilayers formed by the sodium salt of poly(4-styrenesulfonate), PSS, and triblock copolymers of the form PDMAEMA-PCLPDMAEMA (PDMAEMA corresponding to poly[2-( $N, N$-dimethylamino)ethyl methacrylate), and PCL to poly( $\varepsilon$-caprolactone) have been built by layer-by-layer self-assembly from the aqueous polyelectrolyte solutions. Two types of block copolymers have been used which differ

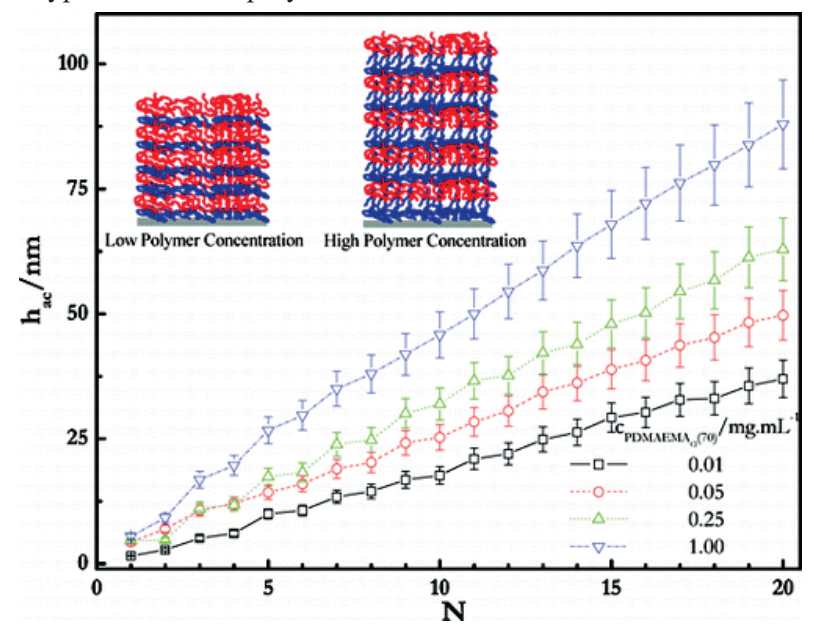

\section{Introduction}

Nanostructured materials are nowadays a fundamental field of research with implications on many applications. ${ }^{1,2}$ Self-assembled multilayers are one of the most versatile and promising systems among this type of materials. In order to construct these multilayers, the layer-by-layer ( $\mathrm{LbL}$ ) technique has been frequently used. In this method charged materials, usually polyelectrolytes, are added sequentially to build up the multilayers by electrostatic interactions. Since the pioneering work of Decher et al., ${ }^{1,3,4}$ the list of possible components has increased enormously: biopolymers ${ }^{5-7}$ (such as peptides, proteins, nucleic acid, etc.), colloidal particles, ${ }^{8,9}$ carbon nanotubes, ${ }^{10}$ and microgels. ${ }^{11}$ Furthermore, the method is not limited to materials with charged groups like strong polyelectrolytes, being possible to build multilayers based on other type of

*To whom correspondence should be addressed. E-mail: rgrubio@quim. ucm.es.

(1) Decher, G.; Schlenoff, J. B. Multilayer Thin Films-Sequential Assembly of Nanocomposite Materials; Wiley-VCH Verlag: Berlin, 2003.

(2) Jaber, J. A.; Schlenoff, J. B. Curr. Opin. Colloid Interface Sci. 2006, 11, 324329.

(3) Decher, G. Science 1997, 277, 1232-1237.

(4) Decher, G.; Hong, J.-D.; Schmitt, J. Thin Solid Films 1992, 210/211, 831-835.

(5) Lvov, Y.; Decher, G.; Sukhorukov, G. B. Macromolecules 1993, 26, 53965399.

(6) Picart, C.; Lavalle, P.; Hubert, P.; Cuisinier, F. J. G.; Decher, G.; Schaaf, P.; Voegel, J. C. Langmuir 2001, 17, 7414-7424.

(7) Boulmedais, F.; Ball, V.; Schwinte, P.; Frisch, B.; Schaaf, P.; Voegel, J. C. Langmuir 2003, 19, 440-445.

(8) Iler, R. K. J. Colloid Interface Sci. 1966, 21, 569-594.

(9) Feldheim, D. L.; Grabar, K. C.; Natan, M. J.; Mallouk, T. C. J. Am. Chem. Soc. 1996, 118, 7640-7641.

(10) Correa-Duarte, M. A.; Kosiorek, A.; Kendulski, W.; Giersig, M.; LizMarzán, L. M. Chem. Mater. 2005, 17, 3268-3272.

(11) Schmidt, S.; Hellweg, T.; von Klitzing, R. Langmuir 2008, 24, 12595-12602.

(12) Burke, S. E.; Barret, C. J. Langmuir 2003, 19, 3297-3303. interactions such as acid-base reactions of weak polyelectrolytes ${ }^{12}$ or hydrogen bonds. ${ }^{13}$ One of the advantages of the LbL method in comparison with other techniques, like Langmuir-Blodgett, ${ }^{14}$ is that the LbL multilayers can be built up on substrates other than flat ones, ${ }^{4}$ e.g., colloidal particles ${ }^{15-17}$ or fluid interfaces (floating multilayers), ${ }^{18,19}$ including liposomes. ${ }^{20}$ The LbL method has been successfully used in many applications and in the design of functional materials as contact lenses, ${ }^{1}$ conductive layers,${ }^{21}$ permselective membranes, ${ }^{22}$ sensors, ${ }^{23}$ light-emitting thin films, ${ }^{24}$ electrochromic films, ${ }^{25}$ nonlinear optical devices, ${ }^{26}$ nanocapsules, ${ }^{27}$ and self-healing coatings. ${ }^{28}$

(13) Sukhishvili, S. A.; Granick, S. J. Am. Chem. Soc. 2000, 122, 9550-9952.

(14) Blodgett, K. B. J. Am. Chem. Soc. 1934, 56, 495.

(15) Caruso, F.; Lichtenfeld, H.; Giersig, M.; Möhwald, H. J. Am. Chem. Soc. 1998, 120, 8523-8534

(16) Sukhorukov, G. B.; Donath, E.; Davis, S.; Lichtenfeld, H.; Caruso, F.; Popov, V. I.; Möhwald, H. Polym. Adv. Technol. 1998, 9, 759-767.

(17) Sukhorukov, G. B.; Donath, E.; Lichtenfeld, H.; Knippel, E.; Knippel, M.; Budde, A.; Möhwald, H. Colloids Surf., A 1998, 137, 253-266.

(18) Guzmán, E.; Ritacco, H.; Ortega, F.; Svitova, T.; Radke, C. J.; Rubio,

R. G. J. Phys. Chem. B 2009, 113, 7128-7137.

(19) Ruths, J.; Essler, F.; Decher, G.; Riegler, H. Langmuir 2000, 16, 8871-8878.

(20) Yaroslavov, A. A.; Rakhnyanskaya, A. A.; Yaroslavova, E. G.; Efimova,

A. A.; Menger, F. M. Adv. Colloid Interface Sci. 2008, 142, 43-52.

(21) Cheung, J. H.; Fou, A. F.; Rubner, M. F. Thin Solid Films 1994, 244, 985989.

(22) Levasalm, J.; McCarthy, T. J. Macromolecules 1997, 30, 1752-1757.

(23) Sun, J. Q.; Wang, Z. Q.; Wu, L. X.; Zhang, X.; Shen, J. C.; Gao, S.; Chi,

L. F.; Fuchs, H. Macromol. Chem. Phys. 2001, 202, 967-973.

(24) Fou, A.; Onitsuka, O.; Ferreira, M.; Rubner, M. F.; Hsieh, B. R. J. Appl. Phys. 1996, 79, 7501-7509.

(25) Laurent, D.; Schlenoff, J. B. Langmuir 1997, 13, 1552-1557.

(26) Laschewsky, A.; Mayer, B.; Wischerhoff, E.; Arys, X.; Bertrand, P.; Delcorte, A.; Jonas, A. Thin Solid Films 1996, 284, 334-337.

(27) De Geest, B. G.; Sukhorukov, G. B.; Möhwald, H. Expert Opin. Drug Delivery 2009, 6, 614-624.

(28) Shchukin, D. G.; Möhwald, H. Small 2007, 3, 926-943. 
Several variables have a strong influence in polyelectrolyte multilayers building process: charge density of the polymer, ${ }^{29,30}$ polymer concentration, ${ }^{31}$ ionic strength, ${ }^{32-34}$ solvent quality for the polyelectrolytes, ${ }^{33} \mathrm{pH}^{35}$ and temperature ${ }^{36}$ are the most relevant. A quantitative understanding of their effect on the structure of the multilayers is crucial, in part because multilayers are nonequilibrium systems, and therefore their structures also depend strongly on the conditions and procedures used for their growth.

Multilayers incorporating copolymers of different charge degree have been studied previously in the literature. ${ }^{29,30,37-39}$ Although in most cases random instead of block copolymers have been used, there are several papers that have described the incorporation of diblock copolymers on the multilayer structure. ${ }^{40-44}$ It is worth to stress that in almost all the studies the copolymers were adsorbed from solutions of a concentration above the critical micellar one (cmc) ${ }^{40-42}$ Even though in the present study all the copolymer solutions will have a concentration well below the $\mathrm{cmc},{ }^{45}$ it is wellknown that in the case of normal surfactants aggregates can be formed at the surface below the cmc. ${ }^{46}$ Recently, Tan et al. ${ }^{47}$ have studied multilayers containing triblock copolymers and used changes of $\mathrm{pH}$ to modify the charge density of the copolymer. In the present paper we will modify the charge density by changing the ratio of the neutral to polyelectrolyte blocks. In this work, multilayers of a polyanion, PSS, and a triblock copolymer with two charged blocks and a central uncharged one will be presented. The effects of the copolymers charge ratio, defined as the relative number of chargeable monomers in the chain, and of the copolymer concentration are explored. Symmetric triblock copolymers of 2-( $N, N$-dimethylamino)ethyl methacrylate and $\varepsilon$-caprolactone have been used as polycations. Two families of copolymers have been prepared. The first family, called $\operatorname{PDMAEMA}_{\mathrm{CI}}(x)$, are hydrochloride copolymers, with $x=90,80$, or $70 \%$ of weight content of the charged monomer 2-( $N, N$-dimethylamino)ethyl methacrylate. The second family, called $\operatorname{PDMAEMA}_{\mathrm{Q}}(x)$, are quaternized with $x=80$ or $70 \%$ of weight content of the charged monomer. ${ }^{45}$ The chemical structures of the copolymers are shown in Figure 1. The interest in the introduction of this kind of copolymers as building blocks in polyelectrolyte multilayers is a consequence of their thermoresponsive behavior and their biodegradability, which open the way for the application of multilayers of this copolymers in

(29) Steitz, R.; Jaeger, W.; von Klitzing, R. Langmuir 2001, 17, 4471-4474. (30) Schoeler, B.; Kumaraswamy, G.; Caruso, F. Macromolecules 2002, 35, 889897.

(31) Saarinen, T.; Österberg, M.; Laine, J. Colloids Surf., A 2008, 330, 134-142.

(32) Schlenoff, J. B.; Dubas, S. T. Macromolecules 2001, 34, 592-598.

(33) Dubas, S. T.; Schlenoff, J. B. Macromolecules 1999, 32, 8153-8160.

(34) Guzmán, E.; Ritacco, H.; Rubio, J. E. F.; Rubio, R. G.; Ortega, F. Soft Matter 2009, 5, 2130-2142.

(35) Zhang, X.; Sun, Y. P.; Gag, M. L.; Kong, X. X.; Shen, J. C. Macromol. Chem. Phys. 1996, 197, 509-515.

(36) Salomaki, M.; Vinokurov, I. A.; Kankare, J. Langmuir 2005, 21, 11232 11240

(37) Voigt, U.; Khrenov, V.; Tauer, K.; Jaeger, W.; von Klitzing, R. J. Phys.: Condens. Matter 2003, 15, S213-218.

(38) Schoeler, B.; Poptoshev, E.; Caruso, F. Macromolecules 2003, 36, 52585264.

(39) Voigt, U.; Jaeger, W.; Findenegg, G. H.; von Klitzing, R. J. Phys. Chem. B 2003, 107, 5273-5280.

(40) Addison, T.; Cayre, O. J.; Biggs, S.; Armes, S. P.; York, D. Langmuir 2008, 24, 13328-13333.

(41) Sakai, K.; Webber, G. B.; Vo, C.-D.; Wanless, E. J.; Vamvakaki, M.; Bütün, V.; Armes, S. P.; Biggs, S. Langmuir 2008, 24, 116-123.

(42) Qi, B.; Tong, X.; Zhao, Y. Macromolecules 2006, 39, 5714-5719.

(43) Wang, Y.; Janout, V.; Regen, S. L. Chem. Mater. 2010, 22, 1285-1287.

(44) Salloum, D. S.; Schlenoff, J. B. Biomacromolecules 2004, 5, 1089-1096.

(45) San Miguel, V.; Limer, A. J.; Haddleton, D. M.; Catalina, F.; Peinado, C.

Eur. Polym. J. 2008, 44, 3853-3863.

(46) Aratono, M.; Kanda, T.; Motomura, K. Langmuir 1990, 6, 843-846.

(47) Tan, W. S.; Cohen, R. E.; Rubner, M. F.; Sukhishvili, S. A. Macromolecules 2010, 43, 1950-1957.

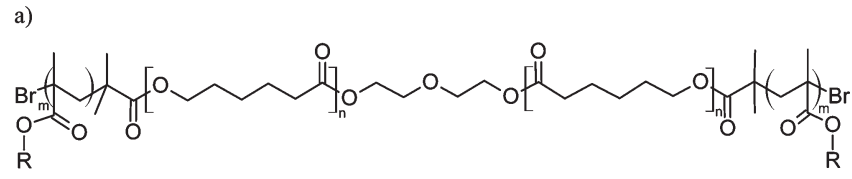

b)<smiles>[Y]C(CC)c1ccc(S(=O)(=O)[O-])cc1</smiles>

Figure 1. Polymers used in the building of the multilayers: (a) $\operatorname{PDMAEMA}(x)$; (b) PSS.

several fields of the bionanotechnology. ${ }^{45,48}$ Moreover, the existence of a hydrophobic central block makes reasonable to expect some aggregation of the PCL blocks after adsorption. Since these blocks are biodegradable, this would open the way to construct highly porous membranes after degradation.

A total of five different copolymers have been studied. This set of copolymers have allowed us to study the effect of the content of amino groups in the polymer chains on the construction of multilayers. The differences in molecular structure between the hydrochloride and the quaternized copolymers have allowed us to analyze the effect of the different degrees of substitution of the amino groups of the chains. This has been possible because the amino groups are tertiary in the case of hydrochloride copolymers, while quarternary ammonium salts in the case of the quaternized copolymers. Results of the dependence of the thickness on the number of layers, for multilayers of the type $\left(\operatorname{PDMAEMA}_{\mathrm{Cl}}(x)+\right.$ $\mathrm{PSS}_{n}$ and $\left(\operatorname{PDMAEMA}_{\mathrm{Q}}(x)+\mathrm{PSS}\right)_{n}$ built up from different conditions, will be presented. The mechanism of multilayer growth, the water content of the films, their mechanical properties, and the kinetics of adsorption of the layers will be also presented.

\section{Experimental Section}

2.1. Chemicals. The polyanion used was poly(sodium 4styrenesulfonate) (PSS), with a molecular weight of $70 \mathrm{kDa}$, from Sigma-Aldrich (Germany). The polycations used were triblock copolymers made of 2-(N,N-dimethylamino)ethyl methacrylate and $\varepsilon$-caprolactone, both in the hydrochloride form $\left(\operatorname{PDMAEMA}_{\mathrm{Cl}}(x)\right)$ with three different charge densities $(x=90,80$, and 70\%) and in the quaternized one (PDMAEMA $\left(\operatorname{PD}_{\mathrm{Q}}(x)\right.$ with two different charge densities $(x=80$ and $70 \%)$ (for details about the used copolymers see Table 1). The triblock copolymers were prepared by ATRP (atom transfer radical polymerization) using a difunctional macromer based on polycaprolactone and varying the degree of polymerization. Selective quaternization of PDMAEMA blocks was carried out by reaction with $\mathrm{HCl}$ and $\mathrm{CH}_{3} \mathrm{I}$ to give rise to water-soluble PDMAEMA $\mathrm{Cl}^{-}$ $(x)$ and $\operatorname{PDMAEMA}_{\mathrm{Q}}(x)$, respectively; details of the synthesis are given in ref 45. The water was of Milli-Q quality (Millipore RG model) with a resistivity higher than $18.2 \mathrm{M} \Omega$ and total organic content (TOC) lower than $10 \mathrm{ppb}$. All the experiments were done at $298.1 \pm 0.1 \mathrm{~K}$. The solutions were prepared by weight using an analytical balance with a precision of $\pm 0.01 \mathrm{mg}$.

The $\mathrm{p} K_{\mathrm{a}}$ of the hydrochloride copolymers in solution has been reported to be $\sim 7.5$. $^{49}$ The $\mathrm{pH}$ of the experiments performed in this work was in the range $(\mathrm{pH} \sim 6-7)$. It has been reported that

(48) San Miguel, V.; Peinado, C.; Catalina, F.; Abrusci, C. Int. Biodeterior. Biodegrad. 2009, 63, 217-223.

(49) Nurmi, L.; Holappa, S.; Nykänen, A.; Laine, J.; Ruokolainen, J.; Seppälä, J. Polymer 2009, 50, 5250-5261. 
Table 1. Details of the Different Copolymers Used in This Study ${ }^{a}$

\begin{tabular}{cccc}
\hline sample & \% weight of charge block & $M_{\mathrm{w}} / \mathrm{Da}$ & $M_{\mathrm{n}} / M_{\mathrm{w}}$ \\
\hline PDMAEMA(90) & 90 & 12800 & 1.22 \\
PDMAEMA(80) & 80 & 12600 & 1.27 \\
PDMAEMA(70) & 70 & 10800 & 1.23
\end{tabular}

${ }^{a}$ The data are for PDMAEMA $\mathrm{PII}_{\mathrm{Cl}}(x)$; for the case of PDMAEMA $\mathrm{PD}_{\mathrm{Q}}(x)$ may be used the same parameters.

the $\mathrm{p} K_{\mathrm{a}}$ of weak polyelectrolytes and surfactants may change on adsorption. ${ }^{50,51}$ Choi and Rubner have described in detail this process as an effect of the decrease of the dielectric constant when the more hydrophobic moieties of the weak polyelectrolytes tend to aggregate. ${ }^{50}$ However, the systems studied by Choi and Rubner are significantly different from the ones presented in this paper because in their case all the monomers hold an ionizable group, and the different hydrophobicity arises from the different chemical structure of the monomers. In the present case, there is a nonionizable central block, PCL, which might present some tendency to aggregate inside the multilayer, thus presenting regions of relatively low dielectric constant. However, there is no reason to expect that the ionizable monomers will be soluble in that hydrophobic regions. In fact, for the copolymers studied in this work, the changes in the degree of ionization of the charged group, as measured by $\xi$-potential of $\mathrm{SiO}_{2}$ particles with a layer of adsorbed copolymers, are in qualitative accordance with the charge ratio. Within one copolymer family, the reduction of the polyelectrolyte/PCL ratio leads to a reduction of the charge. However, the increase of the size of the hydrophobic domains leads to a higher reduction of the charge degree that the one expected taking into account only the polyelectrolyte/PCL ratio. For the same charge ratio the charge density of quaternized copolymers has been found higher than for hydrochloride ones.

2.2. Techniques. A dissipative quartz-crystal microbalance (D-QCM) from KSV (model QCM Z-500, Finland) was used. The quartz crystals, AT-cut, were cleaned with piranha solution $\left(70 \% \mathrm{H}_{2} \mathrm{SO}_{4} / 30 \% \mathrm{H}_{2} \mathrm{O}_{2}\right)$ over a period of $30 \mathrm{~min}$ and then thoroughly rinsed with pure water. The characteristic frequency in vacuum was $f_{0}=5 \mathrm{MHz}$. In order to charge the gold electrode of the quartz crystal, a self-assembled monolayer of the sodium salt of 3-mercaptopropanesulfonic acid was initially built. ${ }^{34}$ D-QCM provided the impedance spectra of the crystal for the fundamental mode and for odd overtones of the fundamental resonance up to the 11 th (central frequency $f_{11}=55 \mathrm{MHz}$ ).

We adopted the model of Johannsmann et al. ${ }^{52}$ to relate the change of the quartz sensor impedance spectra with the adsorbed mass, $m^{*}$, and the shear modulus of the adsorbed film, $\hat{J}$,

$$
m^{*}=m^{0}\left[1+\hat{J}(f) \frac{\rho_{\mathrm{q}}(2 \pi f)^{2} h_{\mathrm{ac}}{ }^{2}}{3}\right]
$$

where $m^{0}$ is the sensed mass obtained by the classical Sauerbrey equation. $^{53}$

$$
m^{0}=-\frac{C \Delta f}{v}
$$

and $\Delta f$ is the frequency change of the maximum of the impedance spectrum after the sensor is in contact with the polymer solution $\left(\Delta f=f_{0}-f\right), v$ is the overtone order $(3,5,7,9$, and 11 in this work), and $C$ is a constant characteristic of the quartz crystal

(50) Choi, J.; Rubner, M. F. Macromolecules 2005, 38, 116-124.

(51) Esker, A. R.; Zhang, L.-H.; Olsen, C. E.; No, K.; Yu, H. Langmuir 1999, 15, $1716-1724$.

(52) Johannsmann, D.; Mathauer, K.; Wegner, G.; Knoll, W. Phys. Rev. B 1992, 46, 7808-7815.

(53) Sauerbrey, G. Z. Phys. 1959, 155, 206-222.

(54) Steinem, C.; Janshoff, A. Piezoelectric Sensors; Springer-Verlag: Berlin, properties $^{54}$ ( $C=17.9 \mathrm{ng} \mathrm{Hz}^{-1} \mathrm{~cm}^{-2}$ in this work). In eq 1 , the real part of $m^{*}$ is the true sensed mass after correcting $m^{0}$ for the viscoelastic character of the adsorbed layer. $\hat{J}(f)$ is the complex shear compliance, $\rho_{\mathrm{q}}$ is the density of quartz $\left(\rho_{\mathrm{q}}=19.3 \mathrm{~g} \mathrm{~cm}^{-3}\right)$, and $h_{\mathrm{ac}}$ is the film thickness obtained using the D-QCM. Since different variables have to be extracted from the impedance spectrum $\left(m^{0}\right.$ and $\left.\hat{J}=J^{\prime}+i J^{\prime \prime}\right)$, it is necessary to measure spectra for several overtones and to assume a constant film density. A typical density value used for polyelectrolyte multilayers is $1.2 \pm$ $0.1 \mathrm{~g} \mathrm{~cm}^{-3} \cdot{ }^{33}$ For the solutions we have taken $\rho_{1}=1 \mathrm{~g} \mathrm{~cm}^{-3}$ and $\eta_{1}=1 \times 10^{-3} \mathrm{~Pa} \mathrm{~s}^{-1}$, as for pure water, because dilute polymer solutions were used. All the experimental spectra were fitted using the Voigt function. ${ }^{55}$

The D-QCM allows one to obtain the relationship between the dissipated and stored energies (during the quartz sensor oscillation) as the dissipation factor, $D . D$ is analogous to the loss tangent on classical rheology experiments.

$$
D=\frac{E_{\text {dissipated }}}{2 \pi E_{\text {stored }}}
$$

where $E_{\text {stored }}$ is the energy stored in the oscillating system, and $E_{\text {dissipated }}$ is the energy dissipated during one period of oscillation.

We have used an imaging null ellipsometer from Nanofilm (model EP3, Germany), and all the experiments have been carried out on a solid-liquid cell at a fixed angle of $60^{\circ}$. Silicon wafers (Siltronix, France) were used as the substrates. ${ }^{34}$ The ellipsometric angles $\Delta$ and $\Psi$ describe the changes in the state of polarization when the light is reflected at a surface. ${ }^{56}$ These angles are related to the ratio of the Fresnel reflection coefficients for the parallel, $r_{\mathrm{p}}$, and perpendicular, $r_{\mathrm{s}}$, light-wave components with respect to the plane of incidence ${ }^{56}$ by

$$
\frac{r_{\mathrm{p}}}{r_{\mathrm{s}}}=\tan \Psi \mathrm{e}^{i \Delta}=\tan \Psi(\cos \Delta+i \sin \Delta)
$$

The uncertainty of $\Delta$ and $\Psi$ were $\pm 0.1^{\circ}$ and $\pm 0.05^{\circ}$, respectively. In order to calculate the adsorbed polymer mass, the refractive index increment was measured with a Brookhaven differential refractometer (model BI-DNDC). ${ }^{57}$ The precision in $(\mathrm{d} n / \mathrm{d} c)_{T}$ was $\pm 0.001 \mathrm{~mL} / \mathrm{g}$. For the calculation of the adsorbed mass, we used De Feijter's equation ${ }^{58}$

$$
\Gamma=\frac{\left(n_{\mathrm{f}}-n_{1}\right) h_{\mathrm{op}}}{(\mathrm{d} n / \mathrm{d} c)_{T}}
$$

where $n_{\mathrm{f}}$ and $n_{1}$ are the refractive index of the film and solvent, respectively, and $h_{\mathrm{op}}$ the film thickness using ellipsometry. The values of $(\mathrm{d} n / \mathrm{d} c)_{T}$ for the different polymer used in this work are collected in the Supporting Information (Table S.I).

In order to evaluate the different effective charge density of the copolymers, we have measured the $\zeta$-potential (Zeta Nanosizer (Malvern Instruments)) of silica particles ( $1 \mu \mathrm{m}$ diameter, SigmaAldrich (Germany)) coated with layers of the different studied copolymers; for the assembling of the coating we have used the method proposed by Sukhorukov et al. ${ }^{19}$ In that way, it is possible to evaluate the different degree of charge overcompensation that produces the different copolymers. The results of $\zeta$-potential of coated particles $(1 \mu \mathrm{m} \mathrm{SiO}$ particles with $\xi=-43 \mathrm{mV})$ are summarized in the Supporting Information (Table S.II). These values have allowed us to order the copolymers as a function of the effective charge density of the chains according to the degree of

(55) Voinova, M. V.; Rodahl, M.; Jonson, M.; Kasemo, B. Phys. Scr. 1999, 59, $391-396$.

(56) Azzam, R. M. A.; Bashara, N. M. Ellipsometry and Polarized Light; Elsevier: North-Holland, 1987

(57) Höok, F.; Kasemo, B.; Nylander, T.; Fant, C.; Scott, K.; Elwing, H. Anal. Chem. 2001, 73, 5796-5804.

(58) De Feijter, J. A.; Benjamins, J.; Veer, F. A. Biopolymers 1978, 17, 17591172 
overcompensation that show the coated microparticles, this parameter being important in order to explain the increase of thickness of the multilayers. ${ }^{50,59}$ The charge density increases as

$$
\begin{aligned}
\text { PDMAEMA }_{\mathrm{Q}}(80) & >\text { PDMAEMA }_{\mathrm{Cl}}(90) \\
& >\text { PDMAEMA }_{\mathrm{Q}}(70) \sim \text { PDMAEMA }_{\mathrm{Cl}}(80) \\
& >\text { PDMAEMA }_{\mathrm{Cl}}(70)
\end{aligned}
$$

\section{Results}

3.1. Multilayer Growth Process. The multilayers were built using a constant PSS concentration of $c \sim 0.5 \mathrm{mg} \mathrm{mL}^{-1}$, while different copolymer concentrations were used to build the different multilayers. In this way we have studied the influence of concentration of PDMAEMA $\mathrm{Pl}_{\mathrm{Cl}}(x)$ and PDMAEMA $\mathrm{PD}_{\mathrm{Q}}(x)$ on the multilayer assembling process. The ionic strength was adjusted in all experiments by addition of $\mathrm{NaCl}\left(c_{\mathrm{NaCl}} \sim 50 \mathrm{mM}\right)$. The adsorption kinetics were followed, until equilibrium, by monitoring the changes in frequency shifts, $\Delta f$, and dissipation factor, $\Delta D$, as well as the ellipsometric angles, $\Delta$ and $\Psi$. Between the adsorption of successive layers, the multilayers were washed with an aqueous $\mathrm{NaCl}$ solution of the same ionic strength as the one used during the adsorption process. In this rinsing process, the polymer chains that are not strongly adsorbed to the multilayer were removed.

Figure 2 shows the central frequency, $f$, of the different overtones (Figure 2a) and the dissipation factor for the third overtone (Figure 2b) for the LbL buildup for the first 10 layers of the multilayer $\left(\text { PDMAEMA }_{\mathrm{Cl}}(70)+\mathrm{PSS}\right)_{n}$. The PDMAEMA $_{\mathrm{Cl}}(70)$ layers shown were adsorbed from an aqueous copolymer solution with a concentration of $0.25 \mathrm{mg} \mathrm{mL}^{-1}$. Similar qualitative results were found for different concentrations and for the different copolymers. Adsorption kinetics experiments were performed under static conditions, thus without stirring. The filling process of the measurement chamber took a few seconds and sometimes leads to an abrupt shift of the signal both in the D-QCM and in the ellipsometry techniques; these fast variations were not used in the analysis of the adsorption kinetics. ${ }^{60}$

In addition to the expected decrease of $f$ as the polymer adsorb onto the quartz sensor, the washout process leads to a small $f$ shift. This shift may be related to the reorganization of polymer chains within the film, either as a consequence of the loss of some weakly bound polymer or due to swelling/deswelling processes of the adsorbed layers. In any case it must be stressed that for all the layers studied in this work the frequency change during the washout process was much smaller than the one found during adsorption. This observation is in agreement with the general view that the electrostatic self-assembly method leads to essentially irreversible adsorption of the oppositely charged polyelectrolyte layers. $^{61}$

The overtone dependence of the frequency shift is related to the mechanical properties of the film. ${ }^{52}$ For purely elastic films (where Sauerbrey equation apply), $\Delta f / v$ for all the overtones should collapse onto a single master curve. However, as it can be observed in Figure 2a, the results obtained is this work show a overtone dependence of $\Delta f / v$, indicating the viscoelasticity of the multilayer and far from the Sauerbrey's limit (eq 2). This observation is further confirmed in Figure $2 b$ by the behavior of the dissipation factor, $D$, that increases strongly during the

(59) Park, S. Y.; Rubner, M. F.; Mayes, A. M. Langmuir 2002, 18, 9600-9604. (60) Viitala, T.; Hautala, J. T.; Vuorinen, J.; Wiedmer, S. K. Langmuir 2007, 23, $609-618$.

(61) Holmberg, K.; Jönsson, B.; Kronberg, B.; Lindman, B. Surfactants and Polymers in Aqueous Solution; John Wiley \& Sons: Chichester, 2002.
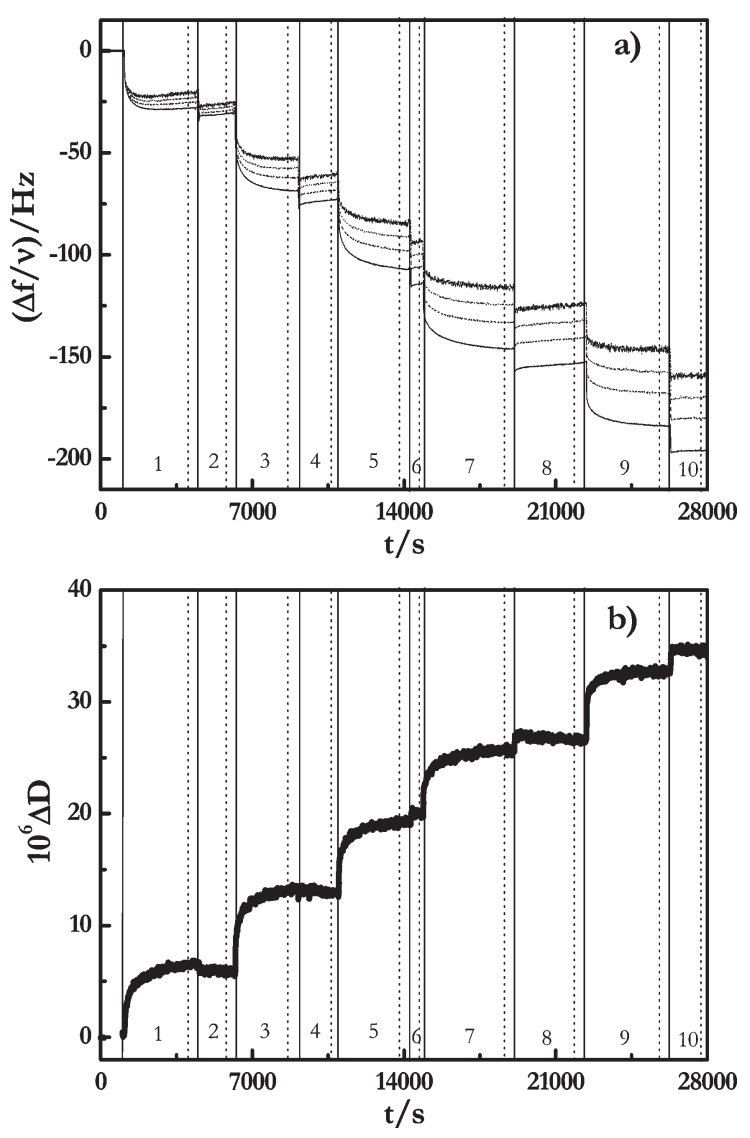

Figure 2. Dissipative quartz crystal microbalance results for the kinetics of adsorption and washing-out process for the first 10 layers of a multilayer (PDMAEMA $\left.\mathrm{Pl}_{\mathrm{Cl}}(70)+\mathrm{PSS}\right)_{n}$ where the PDMAEMA $_{\mathrm{Cl}}(x)$ layers were assembled from solution with $c \sim$ $0.25 \mathrm{mg} \mathrm{mL}^{-1}$. (a) Time dependence of the normalized frequency shifts for different overtones of the quartz sensor, $\Delta f / v$, overtones $v=3(-), v=5(--), v=7(\cdots)$, and $v=9(-\cdot-)$ are shown. (b) Time dependence of the shift in the dissipation factor of the third overtone, $\Delta D$. In both graphs, the vertical continuous lines (-) mark the polyelectrolyte injection and the dashed lines (---) mark the washing with solvent. The number at the bottom of the graph shows the number of the layer that it is adsorbing; odd layers are PDMAEMAA $_{\mathrm{Cl}}(70)$ layers, and even layers are PSS layers.

building process. Under these conditions a fit of the full experimental impedance spectra to the theoretical predictions has to be carried out. ${ }^{52}$ The spectra corresponding to $v=3,5,7$, and 9 have been measured ( $v=11$ was not measured because the signal/noise ratio was small), thus making it possible to calculate the adsorbed mass and the real and imaginary components of the complex shear modulus, $\hat{G}=1 / \hat{J}$, of the polymer layer $(\hat{G}$ was assumed frequency independent in the $15-55 \mathrm{MHz}$ range).

The evolution of the ellipsometric angles, $\Delta$ and $\Psi$, obtained during the growth process leads to similar qualitative conclusions that the D-QCM experiments (an example of the evolution of ellipsometric angles is given in the Supporting Information, Figure S.1).

3.2. Optical, $\boldsymbol{h}_{\mathrm{op}}$, and Acoustic, $\boldsymbol{h}_{\mathrm{ac}}$, Thicknesses. During the multilayer growth process it was found that the frequency shifts (see Figures S.2 and S.3) follow a linear trend as a function of the number of layers, for all the copolymers, independently of the solution concentration or charge ratio, for hydrochloride and quaternized copolymers. The frequency shift for a given layer strongly increases with the copolymer concentration. At low copolymer concentration the data corresponding to different 


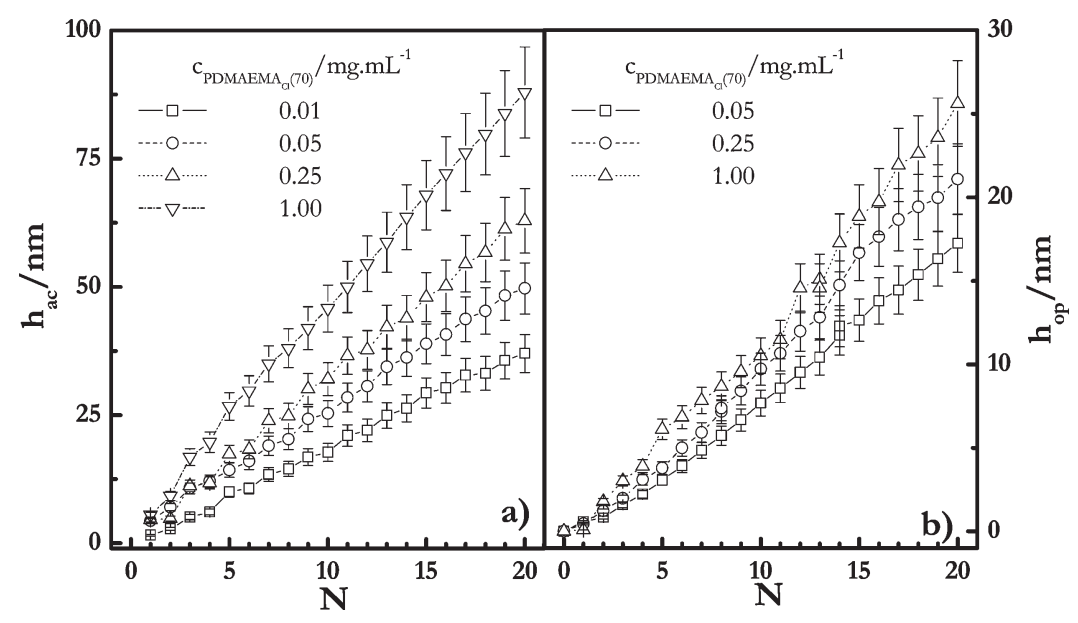

Figure 3. Thickness obtained using D-QCM and ellipsometry for multilayers (PDMAEMA $\left.\mathrm{Cl}_{\mathrm{Cl}}(70)+\mathrm{PSS}\right)_{n}$ built using dipping solutions with different copolymer concentration. (a) D-QCM results and (b) ellipsometric results.

overtones collapse onto a master curve $-\Delta f / v$ vs $N$, which indicates that the film is quite rigid (Sauerbrey's limit). In the case of $\operatorname{PDMAEMA}_{\mathrm{Q}}(x)$ the same qualitative behavior was observed even for higher concentrations (see Figure S.2 in the Supporting Information), ${ }^{52}$ although this is not the case for PDMAEMA $_{\mathrm{Cl}}(x)$ (Figure S.3). This is in agreement with the high dissipation factor values found at high copolymer concentration which suggests that the multilayers are viscoelastic.

The ellipsometry results are in qualitative agreement with the D-QCM results. In order to obtain the ellipsometric thickness, $h_{\text {op}}$, a four-layer model has been used, as in a our previous work. ${ }^{34}$ The analysis of the D-QCM data in terms of eq 1, and of the ellipsometry data in terms of the four-layer model, led to the acoustical, $h_{\mathrm{ac}}$, and to the optical, $h_{\mathrm{op}}$, thickness, respectively, plotted in Figure 3 for the multilayer $\left(\mathrm{PDMAEMA}_{\mathrm{Cl}}(70)+\mathrm{PSS}\right)_{n}$ (other multilayers show similar behavior, see Supporting Information, Figures S.4 and S.5) as a function of the number of adsorbed layers. In all the cases $h_{\mathrm{ac}}>h_{\mathrm{op}}$ due to the fact that D-QCM detects both the polymer and water adsorbed and ellipsometry only the refractive index changes between the multilayer and the solution. The growth process is linear independently of the multilayer studied, and this allows calculating a mean bilayer thickness for the different studied systems.

Figure 4 shows the mean thickness obtained using D-QCM as a function of concentration for the different multilayers of hydrochloride copolymers; the results of both techniques are in good qualitative agreement for all the copolymers studied (for the results of ellipsometry see Figure S.6). It is interesting to compare our results with those in which there is adsorption of micelles on the multilayer. In those systems it has been reported an increase in thickness of 20-30 nm for each layer of micelles, corresponding to the typical micellar diameter. ${ }^{61}$ For our system the micellar diameter is between 20 and $30 \mathrm{~nm},{ }^{45}$ while the layer thickness is lower than $10 \mathrm{~nm}$, for all the multilayers, which allows us to rule out micellar adsorption.

As expected, ${ }^{61}$ the increase in the concentration of the copolymer solution increases the amount of polymer adsorbed. Figure 4 shows that reducing of the charge ratio of the chains for copolymers of the same family, in this case the results for hydrochloride copolymers are shown (qualitatively similar behavior was found for the quaternized copolymers), leads to an increase of the thickness of the layers, in accordance with the results of Voigt et al. ${ }^{37,39}$ for multilayers of PSS and P(DADMAC-stat-NMVA) in the same range of charge ratio of the polymers However, there was found no clear correlation between the thickness of the bilayers obtained using D-QCM

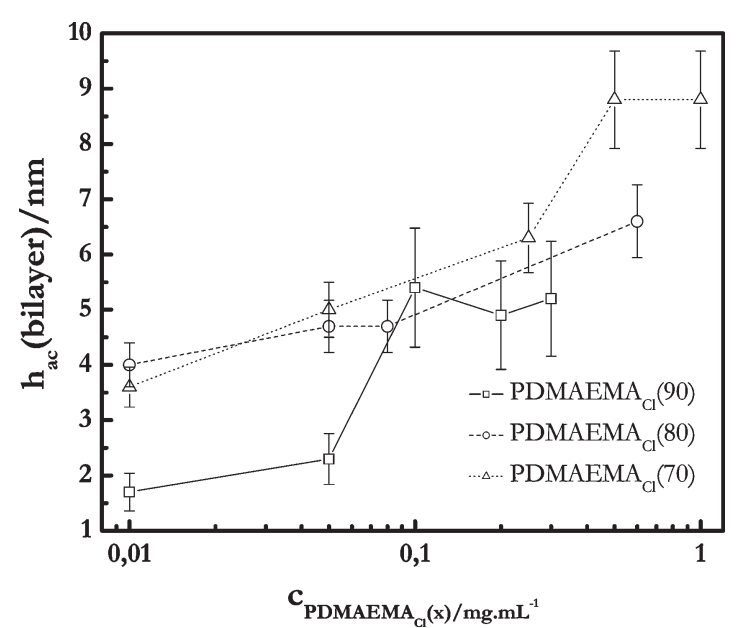

Figure 4. Mean thickness of the bilayers obtained using D-QCM for multilayers (PDMAEMA $\left.\mathrm{Cl}_{\mathrm{Cl}}(x)+\mathrm{PSS}\right)_{n}$ built using copolymer with different charge ratio and varying the concentration of dipping solution of PDMAEMA $\mathrm{Cl}_{\mathrm{Cl}}(x)$, being the PSS concentration fix in all the multilayers $c \sim 0.5 \mathrm{mg} \mathrm{mL}^{-1}$. Legend: $(\square) x=90$; (○) $x=$ $80 ;(\triangle) x=70$.

and ellipsometry when multilayers of PDMAEMA $\operatorname{Pl}_{\mathrm{Cl}}(x)$ and PDMAEMA $_{Q}(x)$ are compared (see Supporting Information Figure S.7). Apparently, different behaviors were obtained from D-QCM and ellipsometry, which might be due to differences on the water content of the multilayers. Considering that the real thickness is close to the optical value, one may conclude that building of multilayers using quaternized copolymers leads to films thicker than those using hydrochloride copolymers. This is in agreement with the results shown by Choi and Rubner (see Figure 7 of ref 50).

3.3. Adsorption Kinetics. In this section, the results corresponding to the kinetics of adsorption for the different layers of copolymers are shown. The adsorption kinetics of the layers for all studied copolymers has been found to be a bimodal process, in accordance with the behavior previously observed for other multilayers. ${ }^{18,34,62-64}$ For the case of the assembling of the PSS

(62) Chiang, C.-Y.; Starov, V. M.; Hall, M. S.; Lloyd, D. R. Colloid J. 1997, 59, 236-247.

(63) Chiang, C.-Y.; Starov, V. M.; Hall, M. S.; Lloyd, D. R. Colloid J. 1997, 59, 236-247.

(64) Raposo, M.; Pontes, R. S.; Mattoso, L. H. C.; Oliveira, O. N. Macromolecules 1997, 30, 6095-6101. 

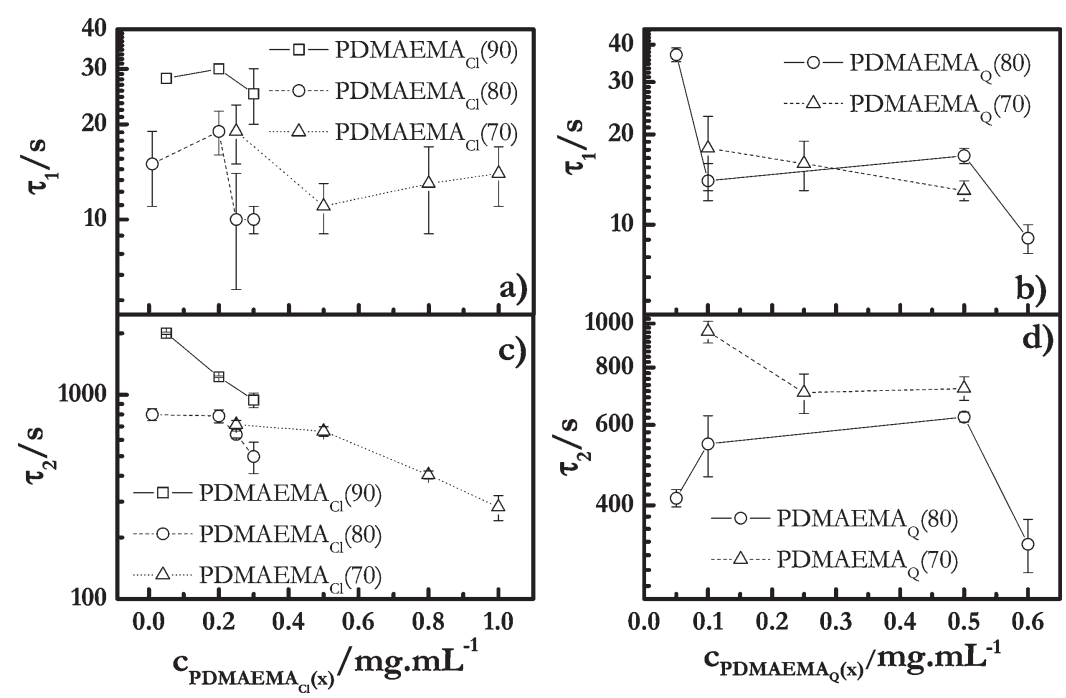

Figure 5. Times for the adsorption of the layer of copolymers with different degree of charge. (a) $\tau_{1}$ for multilayers $\left(\operatorname{PDMAEMA}_{\mathrm{Cl}}(x)+\right.$ PSS $)_{n}$. (b) $\tau_{1}$ for multilayers (PDMAEMA ${ }_{\mathrm{Q}}(x)+$ PSS $)_{n}$. (c) $\tau_{2}$ for multilayers (PDMAEMA Pl $(x)+$ PSS) ${ }_{n}$. (d) $\tau_{2}$ for multilayers $\left(\operatorname{PDMAEMA}_{\mathrm{Q}}(x)+\mathrm{PSS}\right)_{n}$. Legend: $(\square) x=90 ;(\bigcirc) x=80 ;(\triangle) x=70$. Notice that for multilayers $\left(\mathrm{PDMAEMA}_{\mathrm{Q}}(x)+\mathrm{PSS}_{n}\right.$ the charge ratio $x=90$ is not studied.

layers, we did not make the analysis of the kinetic processes because the experimental results showed the existence of complex processes that may be related to the lost of water during the complexation of adjacent layers. This type of behavior was difficult to take account in the kinetic modeling. During the adsorption process, we found a fast adsorption step probably related to the transport of chains to the surface accompanied by a fast mass deposition. This process is not diffusion controlled as revealed by the fact that the mass adsorbed does not show a $t^{1 / 2}$ dependence. The second, slower process may be related to the internal reorganization of the polymer chains in the multilayer. ${ }^{64,65}$ In order to describe the adsorption kinetics, we have used the model proposed by Raposo et al. ${ }^{64}$

$$
\Gamma=A_{1}\left(1-\mathrm{e}^{-t / \tau_{1}}\right)+A_{2}\left(1-\mathrm{e}^{-t / \tau_{2}}\right)^{m}
$$

where $A_{1}$ and $A_{2}$ are the amplitudes and $\tau_{1}$ and $\tau_{2}$ the characteristic times of the fast and slow adsorption steps, respectively. The second term accounted for any reorganization process of the polymer chain after its adsorption at the interface, and it was modeled according to Avrami's model for polymer crystallization. As in a previous work, ${ }^{39}$ all the data obtained in this work are compatible with $m=1$. This allows one to rewrite eq 6 in terms of the maximum surface concentration, $\Gamma_{\infty}$, as

$$
\begin{gathered}
\Gamma_{\infty}=A_{1}+A_{2} \\
\Gamma=\Gamma_{\infty}-A_{1} \mathrm{e}^{-t / \tau_{1}}-A_{2} \mathrm{e}^{-t / \tau_{2}}
\end{gathered}
$$

We have found that for all our adsorption kinetics studied in this work eq 8 describes the experimental data within experimental precision, independently of the polyelectrolyte type and the layer number (an example of the kinetics modeling is shown in the Supporting Information, Figure S.8). This behavior was observed previously for multilayers of (PDADMAC + PSS $)_{n}{ }^{18,34}$

Figure 5 shows the characteristic times of adsorption for both processes in the different multilayers studied. In all the case $\tau_{2}$ is

(65) Lane, T. J.; Fletcher, W. R.; Gormally, M. V.; Johal, M. S. Langmuir 2008, $24,10633-10636$ almost an order of magnitude larger than $\tau_{1}$. The characteristic times for both kinetic processes do not depend on the number of layer for any of the studied systems. This behavior can be understood considering that for the multilayers that grow linearly, like the ones presented in this work, there is not internal reorganization involving the whole structure of the multilayers. Therefore, the adsorption process depends only on the copolymer studied, and hence the kinetics of all the layers in each multilayer are similar. This behavior differs from the one observed for multilayers of $(\mathrm{PDADMAC}+\mathrm{PSS})_{n}$ that present a nonlinear growth for high ionic strengths, where the adsorption time for the reorganization step shows dependence with the layer number. ${ }^{18}$

\section{Discussion}

The results presented above clearly show that the growth and properties of the multilayers studied are strongly influenced by the concentration of the copolymer solution, the content of amino groups on the chains, and the degree of charge of the chains. Table 2 summarizes the effect of the different parameters on the multilayer properties.

4.1. Multilayer Growth. As already mentioned, all the multilayers show a linear growth with the number of layer. The thickness of the layer increases with the copolymer concentration, and it is lower for the hydrochloride copolymers than for the quaternized ones. Furthermore, for a given family of copolymers, the thickness decreases as the charge ratio increases. Besides the thickness of the multilayer, the charge compensation mechanism, the water content, and the mechanical properties of the films are important issues.

The existence of intrinsic compensation implies a stoichiometric 1:1 ratio (polyanion monomer:polycation monomer) in the multilayer, whereas for extrinsic compensation other stoichiometries can be found.

A reasonably way to study the charge-compensation mechanism is to measure the number of PSS monomers adsorbed per charged monomer of the copolymer chain adsorbed in the next layer. For this one can define the compensation ratio, $R_{\mathrm{c}}$, as

$$
R_{\mathrm{c}}=\frac{\rho_{\mathrm{PSS}}}{\rho_{\text {PDMAEMA }}}
$$


Table 2. Resume of the Effect of the Different Parameters Analyzed on the Properties of the Multilayers

\begin{tabular}{lll}
\hline properties & hydrochoride & quaternized \\
\hline
\end{tabular}

Fixed concentration. Effect of increase of amino groups on the chains in

$\begin{array}{lll}\text { thickness } & \text { reduction } & \text { reduction } \\ R_{\mathrm{c}} & \text { increase } & \text { increase } \\ X_{\mathrm{w}} & \text { reduction (for low concentration) } & \text { increasing } \\ & \text { constant (for high concentration) } & \end{array}$

Fixed content of amino groups on the chains. Effect of increase of copolymer concentration in

$\begin{array}{lll}\text { thickness } & \text { increase } & \text { increase } \\ R_{\mathrm{c}} & \text { increase } & \text { increase } \\ X_{\mathrm{w}} & \text { increase (for high content) } & \text { constant (for high content) } \\ & \text { constant (for low content) } & \text { increase (for low content) }\end{array}$

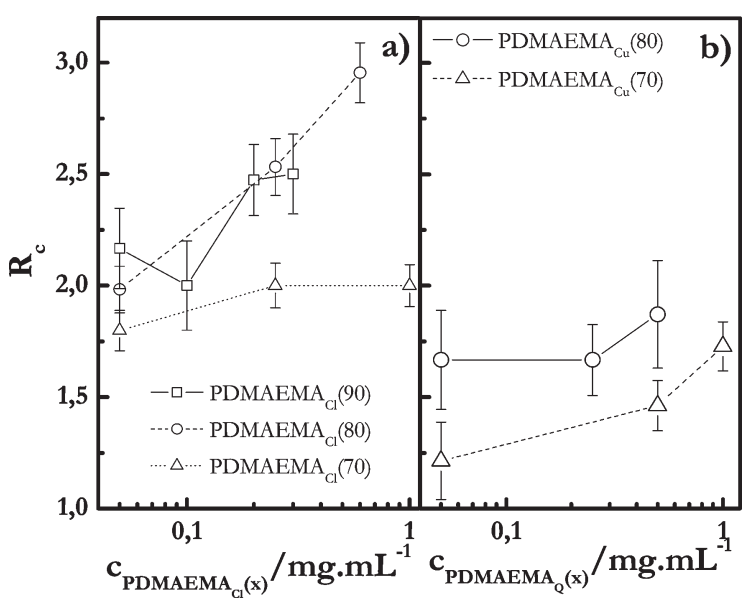

Figure 6. Compensation relations for the different studied multilayers: (a) (PDMAEMA PI $(x)+$ PSS) $)_{n}$ and (b) $\left(\operatorname{PDMAEMA}_{\mathrm{Q}}(x)+\right.$ PSS $)_{n}$. In both graphs: $(\square) x=90$; $(\bigcirc) x=80 ;(\triangle) x=70$.

where $\rho_{\text {PSS }}$ and $\rho_{\text {PDMAEMA }}$ are the monomer surface density of PSS and charge monomer of $\operatorname{PDMAEMA}_{\mathrm{Cl}}(x)$ or $\operatorname{PDMAEMA}_{\mathrm{Q}}(x)$, respectively, for each adsorption cycle. The monomer surface density can be obtained as ${ }^{18,34}$

$$
\rho_{\text {monomer }}=\frac{N_{\mathrm{Av}} \Gamma}{M_{\mathrm{w}}}
$$

where $\Gamma$ is the adsorbed mass obtained using eq $5, M_{\mathrm{w}}$ is the molecular weight of the monomer, and $N_{\mathrm{Av}}$ is Avogadro's number. Notice that $R_{\mathrm{c}}=1$ implies intrinsic compensation and $R_{\mathrm{c}} \neq 1$ implies extrinsic compensation.

The experimental results (see Figure 6) show that for all the multilayers studied the number of PSS monomers is greater than the number of charged monomer of the copolymers; this may be reasonable considering that the conformation of the PSS is typically considered as a random coil, and the effective degree of charge is relatively low (it is necessary to take account that the effective charge on the PSS chains is only $37 \%$ of the nominal one). ${ }^{34}$ The $R_{\mathrm{c}}$ values confirm an extrinsic mechanism in all the studied multilayers. $R_{\mathrm{c}}$ increases with the copolymer concentration for all the systems studied for all the content of amino monomers on the chains.

Polymer chains and counterions adsorb in a hydrated form; thus, the total mass measured by the D-QCM technique includes

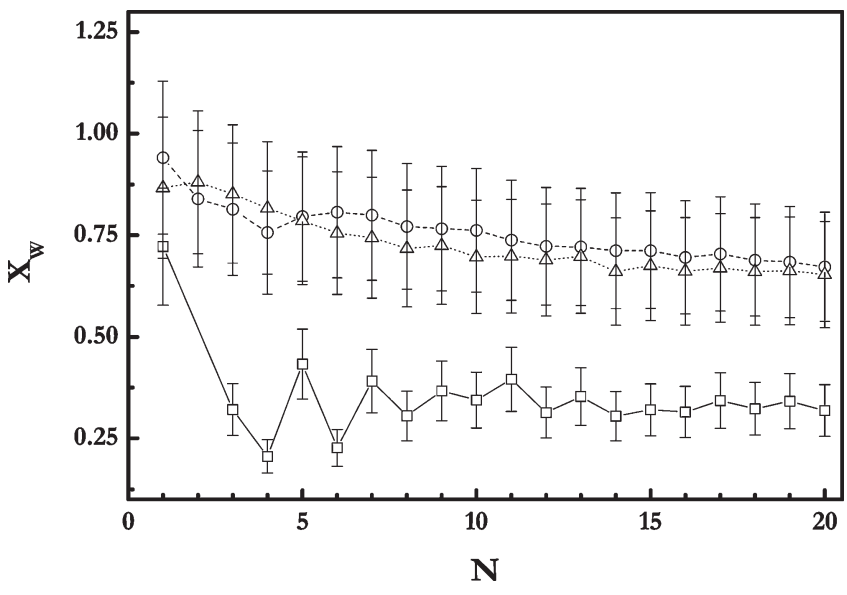

Figure 7. Water contents for different studied multilayers $\left(\text { PDMAEMA }_{\mathrm{Cl}}(x)+\text { PSS }\right)_{n}$ adsorbed from a copolymer concentration of $0.5 \mathrm{mg} \mathrm{mL}^{-1}$. The points are the calculated data, and the lines are only guides for the eyes. Legend: $(\square) x=90$; (O) $x=$ $80 ;(\triangle) x=70$.

the solvent. ${ }^{66}$ The amount of water associated with the multilayers can be obtained by comparing the mass obtained by D-QCM and ellipsometry. ${ }^{34,67}$ In a previous work it has been shown that this method gives results in good agreement with those provided by neutron reflectivity. ${ }^{34}$ The water weight fraction, $X_{\mathrm{w}}$, in the multilayer can be calculated as ${ }^{67}$

$$
X_{\mathrm{w}}=\frac{h_{\mathrm{ac}}-h_{\mathrm{op}}}{h_{\mathrm{ac}}}
$$

The dependence of the water content, $X_{\mathrm{w}}$, with the number of layer is similar to the reported in the literature by us for multilayers of (PDADMAC + PSS $)_{n}{ }^{18,34}$ and by Wong et al. for multilayers of $(\mathrm{PAH}+\mathrm{PSS})_{n} .{ }^{68}$ Figure 7 shows that $X_{\mathrm{w}}$ decreases from values close to 1 for low number of layers until a quasiplateau value $(N \sim 10)$; this qualitative behavior is independent of the system studied in the present work, which suggests that the polymer chains adsorb rather inhomogeneously in the first layers forming isolated adsorbed pancake. ${ }^{69-71}$ These results are in accordance with the model previously reported by us for multilayers of (PDADMAC + PSS $)_{n}{ }^{34}$ and with the structural results obtained by AFM of multilayers by Kujawa et al. ${ }^{72}$ The very high content found for the first adsorbed layer is also in qualitative agreement with the results of Vaccaro et al. ${ }^{69}$

Figure 8 shows $X_{\mathrm{w}}$ for multilayers with 20 layers for all the multilayers studied in this work. It is observed that the water content depends on the copolymers and on the concentration of copolymer. For all the copolymers studied, the water content increases with the polymer concentration. However, the water content shows differences between the hydrochloride and the quaternized copolymers. In the case of $\operatorname{PDMAEMA}_{\mathrm{Cl}}(x), X_{\mathrm{w}}$ depends on the amino content of the chains only for low concentrations of polymer, which may be explained by the low coverage

(66) Vörös, J. Biophys. J. 2004, 87, 553-561.

(67) Halthur, T. J.; Elofsson, U. M. Langmuir 2004, 20, 1739-1745.

(68) Wong, J. E.; Rehfeldt, F.; Hanni, P.; Tanaka, M.; von Klitzing, R. Macromolecules 2004, 37, 7285-7289.

(69) Vaccaro, A.; Hierrezuelo, J.; Skarba, M.; Galletto, P.; Kleimann, J. R.; Borkovec, M. Langmuir 2009, 25, 4864-4867.

(70) Tsukruk, V. V.; Bliznyuk, V. N.; Visser, D.; Campbell, A. L.; Bunning, T. J.; Adams, W. W. Macromolecules 1997, 30, 6615-6625.

(71) Olanya, G.; Iruthayaraj, J.; Poptoshev, E.; Makuska, R.; Vareikis, A.; Claesson, P. M. Langmuir 2008, 24, 5341-5349.

(72) Kujawa, P.; Moraille, P.; Sanchez, J.; Badia, A.; Winnik, F. J. Am. Chem. Soc. 2005, 127, 9224-9234. 


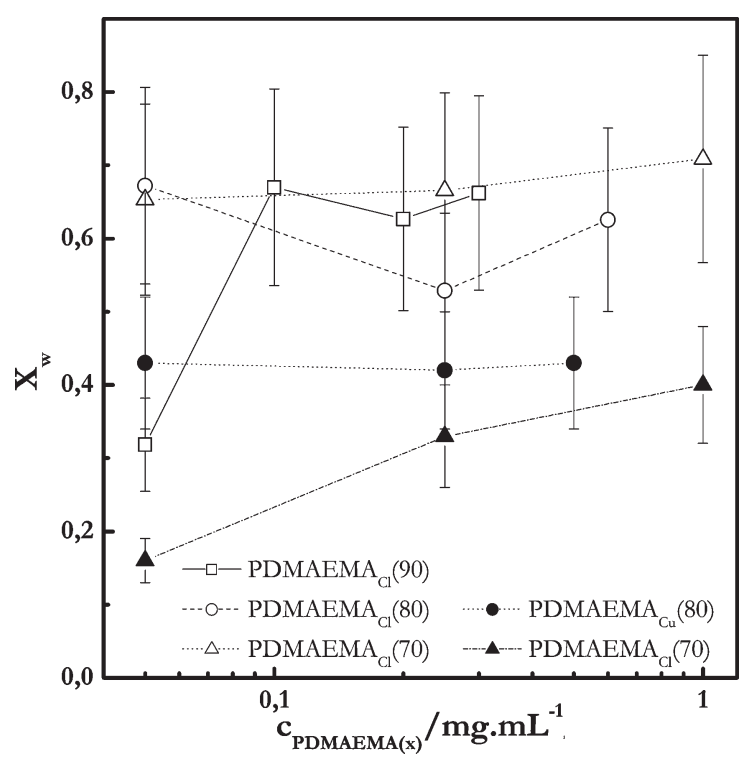

Figure 8. (a) Water content for multilayer of $\left(\operatorname{PDMAEMA}_{\mathrm{Cl}}(x)+\right.$ PSS $)_{n}$ and $\left(\operatorname{PDMAEMA}_{\mathrm{Q}}(x)+\text { PSS }\right)_{n}$ with 20 layers. Legend: $(\square)$ $x=90,(\bigcirc) x=80$, and $(\triangle) x=70$ for hydrochloride copolymers; (๑) $x=80$ and (A) $x=70$ for quaternized copolymers.

that present the layers. The opposite behavior of $\operatorname{PDMAEMA}_{\mathrm{Q}}(x)$ might be explained by considering that the higher effective charge density may increase the repulsion between the adsorbed chains, leading to more inhomogeneous adsorption layers with high water content. No charge ratio dependence of $X_{\mathrm{w}}$ has been observed at high copolymer concentrations for $\operatorname{PDMAEMA}_{\mathrm{Cl}}(x)$.

The small odd-even effect that is observed in Figure 7 may be related to the mechanism of complexation. For the PSS layers, $X_{\mathrm{w}}$ decreases in accordance to the adsorption mechanism observed for PSS layers using D-QCM (see Figure 2), whereas for the layers of copolymers the $X_{\mathrm{w}}$ increases. The oscillatory behavior of $X_{\mathrm{w}}$ has been reported by several authors. ${ }^{34,68,73}$

Finally, the increase of the degree of charge of the chains (comparing hydrochloride and quaternized with the same content of amino groups) leads to a reduction in the water content of the multilayers. This may be due to the stronger interaction between the layers that leads to the formation of more compact layers, with higher amount of adsorbed material, in the case of quaternized copolymers.

The behavior of $G^{\prime}$ and $G^{\prime \prime}$, obtained from the analysis of the D-QCM experiments (see eq 1), was analogous for all the multilayers studied. In all the cases studied, the values of $G^{\prime}$ and $G^{\prime \prime}$ are in the $\mathrm{MPa}$ range, which corresponds to the rubbery region of typical polymers (data are shown in Supporting Information in Figures S.7 and S.8); the values obtained are in all the case in the range of $G^{\prime} \sim 7 \pm 2 \mathrm{MPa}$ and $G^{\prime \prime} \sim 3 \pm 2 \mathrm{MPa}$, giving a $\tan \delta \sim 0.5$ in accordance with the previous results reported for multilayers by Jaber et al. ${ }^{74}$ This is reasonable considering that the multilayers are highly plasticized by water, ${ }^{75}$ and it is in agreement with the results published for other wet multilayers. ${ }^{18,34}$

The multilayers behave as elastic bodies for low concentration of copolymer. However, increasing the concentration reduces the importance relative of the elastic component, $G^{\prime}$, and increases the importance relative of the viscous component, $G^{\prime \prime}$.

(73) McCormick, M.; Smith, R. N.; Graf, R.; Barrett, C. J.; Reven, L.; Spiess, H. W. Macromolecules 2003, 36, 3616-3625.

(74) Jaber, J.; Schlenoff, J. B. Chem. Mater. 2006, 18, 5768-5773

(75) Larsson, R. G. The Structure and Rheology of Complex Fluids; Oxford University Press: Oxford, 1999.
4.2. Adsorption Kinetics. The characteristic time of the fast process, $\tau_{1}$, does not depend on the polymer charge ratio and decreases with increasing concentration as shown in Figure 5.

The characteristic time of the slow process (Figure 5), $\tau_{2}$, decreases with increasing the copolymer concentration. It also decrease as the charge ratio on the chains decreases in the case of hydrochloride copolymers, while the opposite was observed for the dependence of the content in amino groups in the quaternized ones. The effect of the degree of charge of the chains on $\tau_{2}$ depends of the content in amino groups on the chain. For a content of amino groups $x=70$, no dependence was observed; however, for content of amino groups $x=80, \tau_{2}$ decreases in the quaternized with respect of the value obtained in the hydrochloride.

The different effects observed in the reorganizative process may indicate an increase on the number of loops and tails protruding into the solution. This makes easiest and faster the reorganization process.

\section{Conclusion}

The electrostatic self-assembling of PSS and the family of copolymers PDMAEMA $(x)$ (hydrochloride and quaternized) with different charge ratio has been studied by D-QCM and ellipsometry. The effects of the copolymer concentration in the dipping solution, the content of amino groups on the copolymer, and the degree of charge of the chains on the growth of the multilayer have been discussed. Both techniques indicated that the adsorption process was essentially irreversible. This characteristic may be due mainly to the entropy increase associated with the release of counterions during the adsorption process.

The growth mechanism is linear independiently of the conditions used in the assembling of the copolymers used. For all the studied systems the compensation mechanism is extrinsic with participation of the counterions, showing this compensation mechanism dependence with the assembling conditions and with the used copolymer, increasing the degree of extrinsic compensation with the increasing of copolymer concentration in the dipping solution, with the decreasing of the content of amino groups on the chains and with the degree of charge of the chains.

The comparison of the thickness values obtained from D-QCM and from ellipsometry has allowed us to estimate the water content of the multilayer. In general, the overall water content decreases as the number of layers increases, being quite large for films with low number of layers. For films with a large number of layers $(N>10)$, the water content remains almost independent of $N$. For films with a large number of layers $(N>10)$, the water content remains almost constant with $N$ and shows a dependence on the copolymer concentration in the dipping solution, the content of amino groups on the chains, and the degree of charge of the copolymer chains. The dissipative QCM has allowed us to calculate the complex shear modulus of the film thickness. $G^{\prime}$ and $G^{\prime \prime}$ show values in the range of a few MPa, which are characteristic of polymer gels. The importance of the viscous component increases with respect to the elastic component with the increasing of copolymer concentration in the dipping solution; however, the content of amino groups on the chains and the degree of charge do not show effect in the mechanical properties of the multilayers.

In all the studied cases, it was found that the copolymer adsorption followed a bimodal kinetics with a fast process associated with the polymer diffusion to the surface and a slow process associated with the reorganization of the adsorbed polymer chains. We did not found dependence on the adsorption time 
on the number of layers for the different adsorbed layers, in accordance with a linear growth mechanism. The adsorption times for the fast process show dependence only with the concentration of copolymer in solution; however, the adsorption times for the slow reorganizative process show a dependence on copolymer concentration, content of amino groups on the chains, and degree of charge of the copolymers.

The comparative analysis of the results obtained for the hydrochloride and quaternized copolymers allows to conclude that it is not only the charge ratio of the copolymers with amino groups of different nature the parameter that control the adsorption process, existing an important effect of the secondary interaction that can take place in the multilayer building.

Acknowledgment. This work was supported in part by MICINN under grants FIS2009-14008-C02-01, by CAM under grant INTERFASES S05-MAT-227, and by ESA under grant MAP AO00-052. E.G. was supported by a FPU fellowship from MICINN. The authors are grateful to the UIRC of the CAI of Spectroscopy of Complutense University for the use of the ellipsometer. 